\title{
L'approche des éléments culturels dans les publicités pour l'informatique en langue anglaise
}

Janet Atlan

\section{(2) OpenEdition}

\section{Journals}

Édition électronique

URL : http://journals.openedition.org/asp/4074

DOI : 10.4000/asp.4074

ISSN : 2108-6354

\section{Éditeur}

Groupe d'étude et de recherche en anglais de spécialité

\section{Édition imprimée}

Date de publication : 1 décembre 1994

Pagination : 171-176

ISSN : 1246-8185

\section{Référence électronique}

Janet Atlan, «L'approche des éléments culturels dans les publicités pour l'informatique en langue anglaise », ASp [En ligne], 5-6 | 1994, mis en ligne le 09 décembre 2013, consulté le 01 mai 2019. URL : http://journals.openedition.org/asp/4074 ; DOI : 10.4000/asp.4074

Ce document a été généré automatiquement le 1 mai 2019.

Tous droits réservés 


\section{L'approche des éléments culturels dans les publicités pour l'informatique en langue anglaise}

Janet Atlan

\section{L'exercice}

1 Les étudiants doivent choisir une publicité dans une revue informatique et la transformer en publicité uniquement orale. Ils travaillent en groupes de trois et avant de préparer l'oral, ils font un court exercice qui est destiné à leur faire prendre conscience de la structure de la publicité et du langage utilisé (voir annexe 1). Tout l'exercice est fait en classe. La forme finale de la publicité orale est imposée et la publicité produite est corrigée par l'enseignant et répétée avec lui. Ensuite, elle est enregistrée dans le studio son du service audiovisuel de l'IUT de Nancy avec l'aide d'un technicien. Les étudiants peuvent enregistrer leur publicité jusqu'à trois fois et choisir la meilleure version pour la notation. En trois ans nous avons enregistré 46 publicités dont 38 ont été analysées pour ce travail.

\section{Le rôle de l'attitude et des schémas dans la compréhension écrite}

2 Avant de décrire la façon dont nous avons analysé le transfert d'éléments culturels par les étudiants, il faut parler du rôle de l'attitude et des schémas dans la compréhension écrite. Ceux-ci sont impliqués dans la façon dont les étudiants abordent le message culturel contenu dans les publicités. Avant même de travailler, les étudiants ont une attitude préexistante envers la culture représentée par la langue cible. Cette attitude aura un effet sur leur capacité à traiter de nouvelles informations d'ordre culturel qu'ils rencontreront dans les publicités étudiées. 
3 L'attitude est composée de trois éléments (Morgon 1993) : la motivation (les raisons pour l'apprentissage), les croyances (l'interprétation et l'extériorisation que nous faisons du monde qui nous entoure) et les schémas (les connaissances générales d'origine culturelle). Tandis que les croyances sont d'ordre affectif et conscient, les schémas sont d'ordre cognitif et inconscient (ou tout au moins difficilement exprimables).

Carrel (1990) présente trois types de schémas : les schémas de contenu (connaissance du monde et opinions formées à partir de ces connaissances), les schémas formels (connaissances des différents genres de textes) et les schémas linguistiques (connaissances linguistiques). Certaines études montrent une forte influence des schémas de contenu sur la compréhension écrite. La référence aux schémas de contenu pour la compréhension écrite est indicative d'un processus de traitement fondé sur les connaissances (processus haut-bas) ; le lecteur part de ses connaissances générales pour comprendre globalement un texte et non de ses connaissances linguistiques pour le déchiffrer (Carrell \& Eisterhold 1983).

5 Pour cette étude sur la transformation de publicités, nous nous sommes intéressée plus particulièrement à ce type de schémas; car nous pensons que leur influence aura des répercussions sur les éléments culturels qui seront retenus lors de l'exercice de transformation.

6 Carrell (1990) montre aussi l'importance des sous-schémas de contenu communs à chaque discipline d'une langue de spécialité. En effet, dans les publicités spécialisées on peut identifier des schémas de contenu d'ordre général, mais aussi des sous-schémas de contenu informatique déjà connus des étudiants. Il nous semble que ces sous-schémas de contenu dans les publicités informatiques en anglais sont très proches des sous-schémas de contenu dans les publicités informatiques en français. Ils ne sont donc pas source de problèmes de compréhension ou d'assimilation. Par contre, les schémas de contenus sont nouveaux pour les étudiants. La confrontation entre les schémas de contenu des étudiants français et les schémas de contenu dans des publicités en langue anglaise est l'objet de l'analyse que nous avons faite.

7 Les schémas ont un effet de distorsion; le lecteur a tendance à faire correspondre des données nouvelles à ses propres schémas plutôt que d'accepter que ceux-ci soient modifiés. Ceci implique que lors de la création de la publicité orale les étudiants auront tendance à rapprocher l'information culturelle trouvée dans la publicité écrite à leurs propres schémas de contenu plutôt que d'assimiler les schémas de contenu non familiers de celle-ci. Ceci pourrait se traduire par la non-utilisation des éléments des nouveaux schémas de contenu et/ou par un manque de création de nouveaux éléments lors de l'élaboration de la nouvelle publicité.

8 Mais, l'obligation d'utiliser de nouvelles données dans une langue étrangère et dans une activité créative peut amener les étudiants à s'adapter à la culture de la langue cible plutôt que de subir cet effet de distorsion. En effet, la création de ces publicités est une sorte de jeu de rôle, et les jeux de rôle sont utiles pour réduire la dissonance interne face à une nouvelle culture en vue d'un changement d'attitude (Morgon 1993).

9 Un changement d'attitude, et donc des schémas de contenus sous-jacents, ne s'opère pas de façon isolée. Pour qu'il y ait changement, il doit y avoir une restructuration au niveau cognitif qui accompagne un changement affectif. Dans le cas de transformation écrit/oral des publicités, s'il y a création de nouveaux éléments à partir d'éléments existants, cela signifie pour nous qu'il y a eu une confrontation menant à une restructuration cognitive. 
Nous avons donc cherché à mesurer le niveau de créativité dans les transformations de publicités comme indicateur d'une restructuration cognitive menant à une adaptation de schémas de contenu et d'attitude.

\section{Les éléments d'évaluation}

10 Afin d'évaluer le niveau de créativité dans la transformation de publicités, nous avons retenu quatre éléments qui peuvent servir à transmettre un message culturel. Quand ils sont présents dans la publicité d'origine, les étudiants peuvent soit les ignorer, soit les transférer directement, soit s'en servir comme base de création de nouveaux éléments.

D'abord, chaque publicité écrite a un scénario, une idée conductrice dans laquelle s'inscrit la promotion du produit. Elle contient aussi au moins un élément pictural qui met en œuvre le scénario ou montre simplement le produit. Parallèlement à l'élément pictural, il y a le plus souvent dans la publicité des éléments langagiers qui explicitent le scénario ou qui incitent le lecteur à essayer le produit. Enfin, on y trouve parfois un slogan qui peut être repris ou non par les étudiants. Un slogan est une expression plus générale que l'élément langagier. C'est une phrase accrocheuse qui a souvent un rapport avec toute la gamme du fabricant et non seulement avec le produit en question. La figure 1 montre la façon dont nous avons évalué les modifications apportées lors du transfert pour ces quatre éléments dans chaque publicité.

Figure 1 Éléments d'analyse des transferts

\begin{tabular}{|l|l|}
\hline Transfert scénario & Transfert éléments picturaux \\
\hline-1 =pas de scénario & $-1=$ non \\
$0=$ même scénario & $0=$ impossible \\
$+1=$ création de nouveaux éléments & $+1=$ oui, directement \\
$+2=$ création totale & $+2=$ création de nouveaux éléments \\
\hline Transfert éléments langagier & Transfert slogan \\
\hline$-1=$ non, pas de remplacement & $1=$ non \\
$0=$ oui, directement & $0=$ impossible \\
$+1=$ oui, avec nouveaux éléments & $+1=$ oui, directement \\
$+2=$ création totale & $+2=$ création de slogan \\
\hline Barême des transferts & \\
4 à $-2=$ Transfert négatif &
\end{tabular}

$12-1$ à $+3=$ Transfert neutre +4 à $+8=$ Transfert positifll faut noter qu'un transfert négatif n'est pas indicatif de la qualité de la publicité produite, mais décrit simplement la démarche intellectuelle des étudiants.

13 À titre d'exemple d'utilisation des quatre éléments nous montrons le transfert qui a été fait de la publicité pour le scanner Scanman en annexe 2. Le scénario d'origine (comparaison entre l'homme et le singe) a été transformé en faire-part de naissance. 
L'élément pictural (scanners tenus dans les mains) est gardé (« small enough to be held in your hand »). L'élément langagier ("The only competition...requires a much bigger hand ») est conservé avec des changements (« ... as competitive as its bigger cousins »). Le slogan (« Tools that power the desktop ») est utilisé directement. D'après la figure 1, ce transfert obtient une évaluation de +5 et représente un exemple de transfert positif.

\section{L'analyse et les résultats}

Nous avons donc analysé les transferts faits par les étudiants dans chacune des 38 publicités pour lesquelles nous avions à la fois la publicité d'origine et un enregistrement compréhensible. Dans un premier temps, nous présentons trois exemples de transferts de publicités afin de montrer au lecteur ce qui a été analysé comme étant un transfert neutre, un transfert négatif et un transfert positif. Les publicités dont nous parlons se trouvent dans l'annexe 2 .

La publicité pour Verbatim Disks est un exemple de transfert neutre. Le même scénario a été conservé (le désastre de données perdues). L'élément pictural est transféré directement ("I feel like jumping through the window »). L'élément langagier disparaît (sauf pour les deux mots « vital data »). Il n'y avait pas possibilité d'utiliser un slogan et il n'y a pas de création de nouveaux slogans. L'évaluation est donc de 0 et le transfert est neutre.

La publicité pour Acer Computers est un exemple de transfert négatif. Le scénario originel (mise en valeur de la famille) est ignoré et, de plus, il n'y a pas création d'un nouveau scénario. L'élément pictural (l'utilisation du flou pour traduire la vitesse) n'est pas transféré. L'élément langagier (" Speed runs in the Acer family») disparait et le slogan ("Acer - The Word for Value ») n'est pas utilisé. L'évaluation est de -3 et le transfert est négatif.

La publicité pour GEM Artline est un exemple de transfert positif. Le scénario contient de nouveaux éléments par rapport à l'original. En effet, l'expression «Do the Twist » ne fait plus référence à une danse mais devient une façon de manipuler différents objets. Le mouvement impliqué dans l'élément pictural est repris à la fois dans l'intonation, qui est très expressive dans l'enregistrement, et dans les paroles. Il faut noter que dans la version orale de cette publicité, les mots « GEM Artline » sont chantés à chaque reprise. L'élément langagier en rapport avec le scénario est « scale it, rotate it, color it pattern it, mirror it, copy it »; il se retrouve dans le transfert. Il y a aussi création d'une phrase accrocheuse ( Let it turn your head on your PC ») qui fait office de slogan. L'évaluation est de +6 et le transfert est positif.

Toutes les publicités ont été ainsi classées dans une des trois catégories. Un total de 19 transferts était neutre, 9 étaient négatifs et 10 étaient positifs. La figure 2 montre les résultats globaux en pourcentage pour les 38 publicités analysées. 


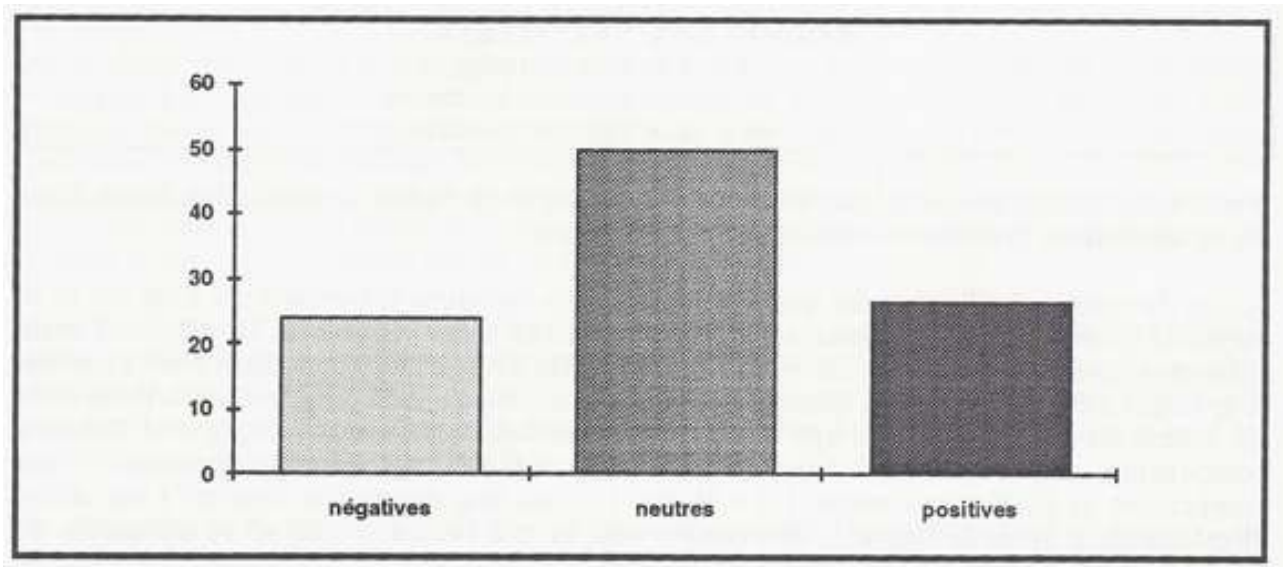

\section{Conclusion}

19 Nous avons constaté que le plus grand nombre de transferts écrit/oral de ces publicités est neutre. Après tout, pour les étudiants, le chemin de moindre résistance pour l'exécution de cet exercice consiste à simplement redire ce qui est dans la publicité d'origine. Mais la nature de l'exercice et de l'analyse permet d'ajouter des éléments, même pour une évaluation neutre. Il aurait été en effet difficile d'éliminer tout élément créatif des transferts neutres. Les transferts dont l'évaluation était neutre ou positive représentent $74 \%$ du total. Ceci signifie, à notre avis, qu'il y a eu confrontation de schémas de contenu dans la plupart des cas. Comme nous l'avons déjà dit, la confrontation peut indiquer une restructuration cognitive et un changement d'attitude. Le changement est, certes, inconscient dans un premier temps, mais il est important dans le cursus des étudiants en langue de spécialité pour lesquels nous visons non seulement une compétence linguistique dans la spécialité mais aussi une prise de conscience culturelle.

\section{BIBLIOGRAPHIE}

Carrell, P. 1990. «Rôle des schémas de contenu et des schémas formels ». Le français dans le monde, numéro spécial: février-mars.

Carrell, P. \& J.C. Eisterhold, J.C. 1983. « Schema theory and ESL reading pedagogy ». TESOL Quarterly 17, 553-573.

Morgon, C. 1993. «Attitude change and foreign language culture learning ». Language Teaching 26/2, 63-75. 


\section{ANNEXES}

\section{Annexe 1 Studying an advertisement}

Before you prepare your oral advertisement, you should find the following information in the written version:

1. Type of advertisement

The public it is aimed at.

The register of language used.

2. The product:

Name of the product

Name of the manufacturer

Generic name (What is it?)

Function (What does it do?)

Price

How to get information on the product

3. Language

Words/expressions that describe the product

Words/expressions that promote the product

Slogans that can be used in your radio ad

4. Form of the advertisement

Your radio ad should be about 30 seconds long and contain the following parts:

Promotion, Description, Information on obtaining the product.

Make sure you pronounce numbers, the names of units (Mhz, lb, etc.) and acronyms correctly. And mind your intonation!

\section{Annexe 2 Transcription des publicités}

\section{SCANMAN SCANNER}

A We are proud to announce the birth of Logitech's scanner model 256 Gray Scale. The latest addition to our family. Small enough to be held in your hand and as competitive as its bigger cousins.

B Capture all the details in your originals in 256 shades of gray. Stitch two images together. Modify image with Ansel Windows-compatible software.

C Get more information from Logitech's customer sales center at 42-21-8699656. Logitech

- tools that power the desktop.

\section{VERBATIM DISKS}

A Oh, God! What a disaster!

B What's wrong with you?

A I've lost vital data. I feel like jumping through the window.

B Before jumping, try Verbatim. And life will be wonderful.

C Save time and money with Verbatim dicks. The safest in the world. Protected by Teflon coating, making them reliable. You won't lose data.

B Verbatim uses laser technology 
C It's certified $100 \%$ error-free.

B So next time, before jumping, insist on Verbatim.

\section{ACER COMPUTERS}

A You need a fast and powerful computer?

B From general business to complex engineering and software development applications, the Acer 1100 family is the answer.

C The exclusive architecture gets your work done faster. Cache memory, disk cache, multiple bus master and more.

B Moreover, each Acer 1100 computer is compatible with industry standard software and hardware.

A To find out more about how the Acer 1100 family puts your computing needs in perspective, contact any of Acer's 10,000 dealers around the world.

\section{GEM ARTLINE SOFTWARE}

A Can I twist my face without turning a hair?

$B$ Yes, you can do it.

C GEM Artline.

A Can I turn a caterpillar into a butterfly?

$B$ Yes, you can do it.

C GEM Artline.

A Can I twist the nose of my neighbor without holding it?

$B$ Yes, you can do it.

C GEM Artline.

A Can I scale it?

B Uh-huh

A Rotate it?

B Uh-huh

A Color it?

B Uh-huh

A Pattern it? Mirror it? Copy it?

B Yes, you can do it.

C GEM Artline

B With the new Artline graphic illustrator. Artline, the ideal design tool for the most innovative high-quality texts and graphics.

C GEM Artline. Let it turn your head on your PC.

B For more information, contact your nearest dealer. Or call 8-00 -4 43-4200.

\section{RÉSUMÉS}

Cet article a pour objet d'étudier l'approche par des étudiants français d'éléments culturels contenus dans des publicités informatiques en anglais. Il est fondé sur un exercice de transformation de publicité écrite en publicité orale fait avec des étudiants en deuxième année d'IUT Informatique. La méthode que nous avons utilisée pour évaluer le transfert d'éléments culturels par les étudiants est décrite et les résultats de l'analyse nous permettent de constater qu'il y a probablement une prise de conscience culturelle de la part des étudiants lors de l'exercice. 
The aim of this article is to study French students' reaction to cultural elements contained in computer advertisements in English. It is based on an exercise done with second-year students in a French technical college which consists in transforming written advertisements to spoken versions. The method used to evaluate the transfer of cultural elements is described and the results of the analysis allow us to postulate that the students become more culturally aware during this exercise.

INDEX

Mots-clés : anglais de spécialité, attitude, culture, schéma

Keywords : attitude, culture, ESP, schema

\section{AUTEUR}

\section{JANET ATLAN}

Janet Atlan enseigne àl'IUT de Nancy 2. Elle est membre de l'équipe COSTECH-UTC.

janetatlan@gmail.com 